\title{
Differential Impact of HLA-B*44 Allelic Mismaches at Position 156 on Peptide Binding Specificities and T-Cell Diversity
}

\section{Soumya Badrinath ${ }^{1}$, Trevor Huyton ${ }^{1}$, Heike Kunze-Schumacher ${ }^{1}$, Holger Andreas Elsner ${ }^{2}$, Rainer Blasczyk ${ }^{1}$ and Christina Bade-Doeding ${ }^{1 *}$}

${ }^{1}$ Institute for Transfusion Medicine, Hannover Medical School, Hannover, Germany

${ }^{2}$ Laboratory Dr. Fenner and collueges, Hamburg, Germany

\begin{abstract}
The molecular understanding of how we can mismatch patients and donors and still have successful clinical outcomes will help to guide the future of unrelated bone-marrow transplantation.

Single amino acid mismatches at position 156 on the alpha 2 helix of $B^{*} 44$ variants have been described to cause immunological episodes. The magnitude of permissivity between B44/156 variants differs from peptide presentation independent of the peptide loading complex ( $\left.B^{*} 44: 28\right)$ to influencing clinical episodes ( $B^{*} 44: 02$ vs. $\left.B^{*} 44: 03\right)$. We here investigated if the single exchange of an Asp>Glu as occurring in $B^{\star} 44: 35$ at residue 156 would force an immune response in vitro.
\end{abstract}

We developed an in vitro system by recombinant co-expression of a single membrane-bound allogeneic HLA class I molecule in donor cells and co-incubating these cells with autologous T-cells. This strategy enables the study of single HLA class I mismatches and excludes the influence of minor antigens. We found these T-cells to be able to differentially discriminate between mismatched $B^{*} 44$ subtypes and their micropolymorphism. To understand how certain $\mathrm{pHLA}$ landscapes shaping the alloreactive immune response, we sequenced the individual peptides derived from $B^{*} 44 / 156$ subtypes using LC-ESI-MS/MS technology. Based on the peptide data we modeled the structure of the $B^{*} 44: 35$ variant and can describe the unexpected immunological reaction of the mismatched $B * 44$ subtypes through structural manipulation of the heavy chain.

The meticulous characterization of peptide-binding profiles for key alleles, as well as the evaluation of T-cell responses and structural analysis in the context of one-allele mismatches will open the door to a new era in bonemarrow transplantation.

Keywords: HLA-polymorphism; Haematopoetic stem cell transplantation; Peptide binding motif

Abbreviations: HLA: Human Leucocyte Antigens; mHAGs: Minor Histocompatibility Antigens; HSCT: Hematopoietic Stem Cell Transplantation; GvHD: Graft versus Host Diseasel; PBR: Peptide Binding Region; AA: Amino Acid

\section{Introduction}

Allogeneic hematopoietic stem cell transplantation (HSCT) is used as a therapeutic strategy for treating many haematological malignancies and certain other immune related disorders. Selection of donors for HSCT involves rigorous assessment and matching of human leukocyte antigen (HLA) types between the donors: recipient pair. The best outcomes following transplantation have been achieved when an HLA identical sibling donor is used for transplantation. However, there is to date still only a $30 \%$ chance of finding such a donor and in most instances HLA mismatched stem cells from related or unrelated donors are used for transplantation [1]. Such kinds of transplants are therefore often associated with severe graft-versus-host-disease (GVHD) and transplant rejection [2]. Many clinical studies have demonstrated that the effect of HLA mismatches depends on the nature of exchanged amino acids (AA) and their location within the heavy chain of the class I molecule [3]. As of today, there is no strategy available for the selection of the less permissive class I mismatch when no identical donor but multiple mismatched donors are available due to the enormous degree of HLA class I polymorphisms (n) and the quantity of possible mismatches [magnitude $=1 / 2 \cdot n \cdot(n-1)$ ] that can occur.

Foreign tissue is recognized through several mechanisms, involving direct and indirect recognition of HLAs and/ or minor histocompatibility antigens (mHags) [4-6]. Retrospective analysis of donor: recipient pairs have demonstrated that the strongest effects following transplantation are HLA-dependent [4-7]. Alloreactivity following HSCT from HLA-identical donor:recipient pairs can be attributed to the immunological effect drived from mHAGs, whereas that following HSCT from HLA mismatched unrelated donors is caused by the cumulative effects of both HLA mismatching and mHags (Table 1). In such a scenario, the magnitude of HLA mismatch effect alone remains unclear and is extremely difficult to separate out from the enormous number of potential mHags present in the human genome.

There is growing evidence highlighting the impact of single AA polymorphisms occurring at certain key residues within the class I molecules in influencing post-transplantation outcomes. One such polymorphism occurs at position 156 , located at the center of $\alpha 2$ helix within the HLA-class I heavy chain and found to be important in binding of peptides at positions $3,4,5,6$ and/ or 7 [8]. We observed that

*Corresponding author: Dr. Christina Bade-Doeding, Institute for Transfusion Medicine, Hannover Medical School Medical Park, Feodor-Lynen-Str. 21 30625 Hannover, Germany, Tel: +49 511532 9744; Fax: +49 511532 9701; E-mail: bade-doeding.christina@mh-hannover.de

Received February 27, 2014; Accepted April 03, 2014; Published April 05, 2014

Citation: Badrinath S, Huyton T, Kunze-Schumacher H, Elsner HA, Blasczyk R, et al. (2014) Differential Impact of HLA-B*44 Allelic Mismaches at Position 156 on Peptide Binding Specificities and T-Cell Diversity. J Stem Cell Res Ther 4: 192 doi:10.4172/2157-7633.1000192

Copyright: () 2014 Badrinath S, et al. This is an open-access article distributed under the terms of the Creative Commons Attribution License, which permits unrestricted use, distribution, and reproduction in any medium, provided the original author and source are credited. 


\begin{tabular}{|l|l|l|l|}
\hline HLA allele & Non-HLA allele & $\begin{array}{l}\text { Donor-Recipient } \\
\text { relation }\end{array}$ & Clinical situation \\
\hline identical & identical & syngenic & Monozygotic twins \\
\hline identical & different & allogenic & Related or unrelated donors \\
\hline different & different & allogenic & Unrelated donors \\
\hline Different & identical & allogenic & artificial \\
\hline
\end{tabular}

Table 1: Comprehensive outlook of different clinical transplantation scenarios. Characteristics of allogeneic incompatibilities for various donor: recipient constellations in a HSCT situation. In a clinical transplantation setting where related/ unrelated donors are considered for transplantation, HLA alleles remain either identical/ different while the MHAGs are always different among the donor: recipient pair and its effects must be taken into account. The HLAdifferential and mHAG-identical situation does not occur, and therefore in a natural setting, it is not possible to determine the HLA mismatch effects exclusively. The lower line emphasizes the characteristics of the artificial allogeneic system, where the magnitude of HLA mismatch effects can be determined independent of $\mathrm{mHAG}$ effects.

within the HLA-A ${ }^{\star} 24, B^{\star} 35$ and $B^{\star} 44$ groups there are selected alleles that differ exclusively by an AA exchange at position 156 . Interestingly these "micropolymorphisms" are able to alter the magnitude of permissivity between the $\mathrm{B}^{\star} 44$ subtypes by selecting and presenting an alternate peptide repertoire independently of the peptide loading complex ( $\left.B^{\star} 44: 28^{156 \mathrm{Arg}}\right)$ [9]. This lack of permissivity has also been highlighted in clinical episodes (e.g $B^{\star} 44: 02^{156 \mathrm{Asp}} \mathrm{v} / \mathrm{s}^{\star} 44$ :03 $3^{156 \mathrm{Leu}}$ ) [10]. Another naturally occuring variant found within the $\mathrm{B}^{\star} 44$ group is the $B^{\star} 44: 35^{156 \text { Glu }}$ allele. Given this conservative substitution at position 156 between $\mathrm{B}^{\star} 44: 02^{156 \mathrm{Asp}}$ and $\mathrm{B}^{\star} 44: 35^{156 \mathrm{Glu}}$ (i.e maintaining an acidic AA at position 156) we wanted to determine if the Asp to Glu exchange influences the immunological functions and results in alloreactivity in vitro between $\mathrm{B}^{\star} 44: 02^{156 \mathrm{Asp}} \mathrm{v} / \mathrm{s} \mathrm{B}^{\star} 44: 35^{156 \mathrm{Glu}}$ and compare the observed results with the well characterized $B^{\star} 44: 02^{156 \mathrm{Asp}} \mathrm{v} / \mathrm{s} B^{\star} 44: 03^{156 \mathrm{Leu}}$ alloreative responses observed during clinical transplantation scenarios.

Currently, there is no reliable method that can be used for selective evaluation of allogenicity due to single mismatches within the HLA class I molecule and to distinguish their effects from the influence of mHAGs. We therefore developed an in vitro system that we termed "the artificial allogeneic system" (Figure 1). The basic principle of our in vitro model is that autologous donor cells from HLA-B ${ }^{\star} 4402$ positive donors are transduced with lentiviral constructs encoding for an additional membrane-bound allogeneic HLA class I molecule (Figure 2), in this case HLA-B ${ }^{\star} 44: 03$ or $\mathrm{B}^{\star} 44: 35$. The recombinant alloHLA molecule displayed in the model therefore the sole alloreactive feature. We wanted to determine if the observed allogenicity if any is reflected on the peptide binding features exhibited by these allotypes. The outcome following transplantation is to a large extent influenced by the T cell recognition of class I alleles as self/ antigenic [11] and $\mathrm{T}$-cell recognition of these different alleles is in turn influenced by the ability of these variants to bind peptides with distinct AA sequences [12]. Therefore, we can define histocompatibility based on the repertoire of peptides that are selected and presented by these different allotypes. Our aim was to integrate the data obtained from mass spectrometric sequencing of endogenous peptide repertoire presented by these polymorphic variants along with observed $\mathrm{T}$ cell responses in order to determine permissible/ non-permissible mismatches for transplantation within the $\mathrm{B}^{\star} 44$ allelic group. This strategy is likely to be a highly valuable instrument for the validation of computational allogenicity prediction tools.

\section{Materials and Methods}

\section{Designing lentiviral vectors}

cDNA from a HLA-B ${ }^{\star} 4402$ positive donor (exons 1-7) was amplified by PCR using the primers HLA-B1-TAS (5 GAGATGCGGGTCACGGCG $\left.3^{\prime}\right)$ and HLA-B-TAAS-E7 (5' TCAAGCTGTGAGAGACACATCAG $3^{\prime}$ ). The PCR product was

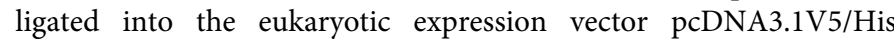
using the pcDNA3.1V5/His TA-cloning Kit (Invitrogen, Karlsruhe, Germany). The $\mathrm{B}^{\star} 4402$ insert was ligated into the pRRL.PPT.SFFV. mcs.pre vector and the insertion verified by sequencing (forward and reverse direction) using an ABI PRISM 377 sequencer (Applied Biosystems, Foster City, CA, USA). HLA-B ${ }^{\star} 44: 03$ and $B \star 44: 35$ variants with a Leu and Glu respectively at position 156 were generated by site directed mutagenesis (QuickChange Multi Site-Directed Mutagenesis Kit, Stratagene, Amsterdam, The Netherlands) using the pcDNA3.1V5/ His $/ \mathrm{B}^{\star} 44: 02$ vector as the template. The $\mathrm{B}^{\star} 44$ inserts were ligated into the pRRL.PPT.SFFV.mcs.pre vector and ligation was verified by sequencing as described earlier.

Soluble HLA-B ${ }^{\star} 44: 03$ and $B^{\star} 44: 35$ variants were generated from pRRL.PPT.SFFV.mcs.pre/ $\mathrm{B}^{\star} 44$ vectors by introducing a stop codon after exon 4 by site-directed mutagenesis performed as described earlier.

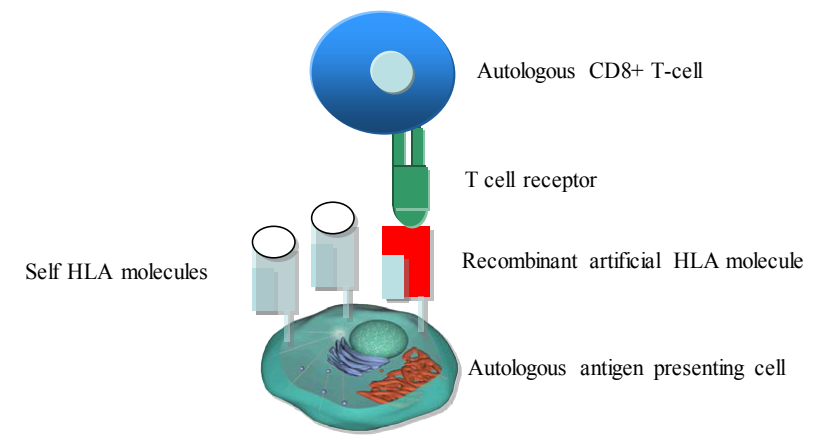

Figure 1: Principle of the artificial allogeneic system. DCs cultivated from peripheral blood monocytes are transduced with an extra HLA allele in addition to the endogenous HLA alleles expressed by the donor. The transduced recombinant HLA molecule is the only allogeneic feature and the observed T-cell alloreactivity can be directly attributed towards the recombinant allogeneic HLA molecule.

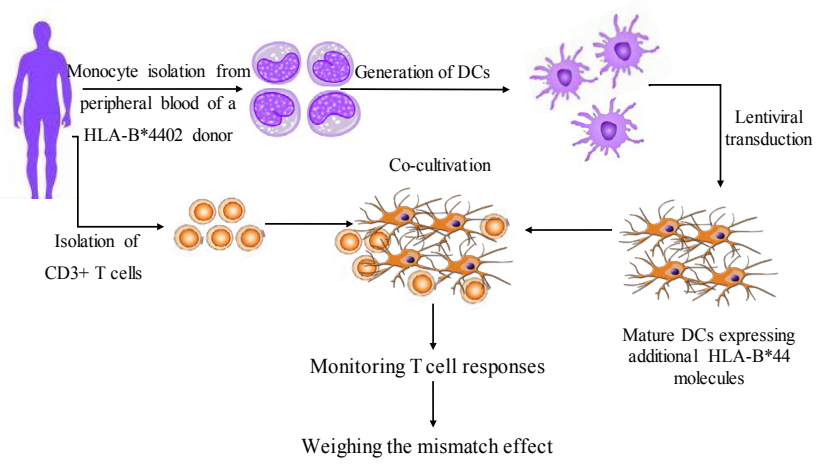

Figure 2: Strategy for the detection of immune responses against an artificial allogeneic HLA variant. Experimental outline for detecting the T cell response against an individual HLA class I molecule. Monocytes isolated from peripheral blood of a selected donor (here expressing HLA-B*44:02) are used for the generation of DCs. Mature DCs are transduced with lentiviral constructs encoding for an additional full length HLA-B* 44 allele differing by a single AA at position 156. These DCs are co-cultured with freshly isolated autologous CD3+ T cells. The HLA mismatch effect is determined by monitoring the responses of the stimulated $T$ cells using recombinant DCs expressing addition HLA-B*44 molecule as target cells. 


\section{Production of lentiviral particles in HEK 293T cells}

$6 \times 10^{6}$ HEK293T cells $/ 10 \mathrm{~cm}$ dish were plated out along with $5 \mathrm{ml} \mathrm{DMEM} / 10 \%$ FCS media (+G418 $100 \mu \mathrm{g} / \mathrm{ml})$. Lipofectamine (Invitrogen) was added dropwise into a $15 \mathrm{ml}$ falcon tube containing OPTI-MEM (Gibco, Life Technologies GmbH, Darmstadt, Germany) and incubated for $5 \mathrm{~min} .10 \mu \mathrm{g}$ of transfer vector (pRRL HLA-B ${ }^{\star} 44: 02$ or HLA-B ${ }^{\star} 44: 03$ or $\left.B^{\star} 44: 35\right)$ packaging plasmid: $5 \mu \mathrm{g}$ psPAX2 and envelope plasmid: $5 \mu \mathrm{g}$ pMD2G were added to another $15 \mathrm{ml}$ falcon tube containing OPTI-MEM. The lipofectamine-OPTI-MEM mixture was added dropwise into falcon tube containing the plasmids, mixed and incubated for $20 \mathrm{~min}$ before adding to the HEK293T seeded plates. The media was changed $8 \mathrm{~h}$ post transfection. After $24 \mathrm{~h}$, the supernatant containing viral particles was collected and filtered through a $0.45 \mu \mathrm{m}$ filter (Millipore $\mathrm{GmbH}$, Schwalbach, Germany) and concentrated by centrifuging overnight at $16^{\circ} \mathrm{C}$ at $10,000 \mathrm{rpm}$. Lentiviral pellet obtained was dissolved in RPMI/ $2 \%$ AB-sera (Invitrogen; c.c. pro, Oberdorla, Germany).

Lentiviral particles for production of soluble HLA class I molecules were produced by transfecting $5 \times 10^{6}$ HEK293T cells with $10 \mu \mathrm{g}$ of transfer vector ( $p R R L$ sHLA- $B^{\star} 44: 03$ or sHLA-B $^{\star} 44: 35$ ) packaging plasmid: $5 \mu \mathrm{g}$ psPAX2 and envelope plasmid: $5 \mu \mathrm{g}$ pMD2G. Following media exchange, supernatant containing lentiviral vector was collected, filtered and concentrated by centrifuging overnight at $16^{\circ} \mathrm{C}$ at 10.000 $\mathrm{rpm}$. The lentiviral pellet was resuspended in RPMI 1640 medium supplemented with 10\% FCS and L-glutamine (Invitrogen).

\section{Cultivation, maturation and transduction of dendritic cells}

PBMC donors used in this study were all 4 digit HLA typed for HLA- $A$ and $B$ alleles. The donors were HLA- $A^{\star} 02: 01, A^{\star} 32: 01, B^{\star} 07: 02$, $\mathrm{B}^{\star} 44: 02$ / HLA-A $\mathrm{A}^{\star} 01: 01, \mathrm{~A}^{\star} 68: 01, \mathrm{~B}^{\star} 08: 01, \mathrm{~B}^{\star} 44: 02 / \mathrm{A}^{\star} 01: 01, \mathrm{~A}^{\star} 25: 01$, $B^{\star} 08: 01, B^{\star} 44: 02$. PBMCs were isolated from the blood of these healthy platelet donors by density gradient centrifugation. Freshly isolated blood (not older than $8 \mathrm{~h}$ post donation) was diluted with 2-4 volumes of PBS. The diluted cell suspension was added dropwise over $15 \mathrm{ml}$ of leucosep (Greiner Bio-One, $\mathrm{GmbH}$ ) in a $50 \mathrm{ml}$ falcon tube and centrifuged at $2000 \mathrm{rpm}$ for $15 \mathrm{~min}$ at room temperature without breaking. Following centrifugation, the upper plasma layer was aspirated carefully leaving behind the interphase containing mononuclear cell layer (lymphocytes, monocytes and thrombocytes) undisturbed. The mononuclear cell layer was resuspended in $15 \mathrm{ml}$ of PBS and centrifuged at $1400 \mathrm{rpm}$ for $15 \mathrm{~min}$. The cell pellet was dissolved in $25 \mathrm{ml}$ of PBS and centrifuged at $800 \mathrm{rpm}$ for $15 \mathrm{~min}$. The supernatant was discarded and the cell pellet was resuspended in RPMI/ $2 \% \mathrm{AB}$-sera and the cell number was determined. On day 0 , freshly isolated PBMCs supplemented with RPMI/ $2 \%$ AB-sera were plated on falcon cell culture dishes (BD Biosciences), precoated with Sandoglobulin $(30 \mu \mathrm{g} / \mathrm{ml})$ (Sandoz, Holzkirchen, Germany) and incubated for $1-1.5 \mathrm{~h}$ in a humidified $37^{\circ} \mathrm{C}$ $\mathrm{CO}_{2}$ incubator. Monocytes were adherent at this stage, remaining nonadherent cells in the supernatant have been removed. The monocytes were cultured in RPMI/ 2\% AB-sera (Invitrogen; c.c.pro) over night. On day 1 , monocyte containing media was removed, filtered and stored at $-20^{\circ} \mathrm{C}$. Monocytes were cultured in fresh RPMI/ $2 \% \mathrm{AB}$-sera supplemented with IL-4 (500 U/ ml) (PeproTech GmbH, Hamburg, Germany) and GM-CSF (800 U/ ml) (PeproTech GmbH, Hamburg, Germany). On day 4 if the cells were still adherent, media was removed completely and fresh RPMI/ 2\% AB-sera supplemented with IL-4 (500 $\mathrm{U} / \mathrm{ml})$ and GM-CSF (800 Uml) were added to the cells. If the cells were in suspension, additional media along with the cytokines for the entire volume was added. On day 5 , the supernatant containing mature dendritic cells (DCs) was collected and centrifuged at $800 \mathrm{rpm}$. The cells were counted and plated at a density of $1 \times 10^{6}$ cells per well in a 6 well cell culture plate supplemented with media containing the cytokines. On day 6 , the monocyte conditioned media (from day 1 ) was added to the cells. Mature DCs were transduced with lentiviral vectors on day 7. The lentiviral transduction of the DCs was performed adding the dissolved lentiviral-pellet in the presence of $8 \mu \mathrm{g} / \mathrm{mL}$ protamine sulfate (Sigma-Aldrich) to the DC plates followed by incubation for $8 \mathrm{~h}$. Transduced DCs were removed from the plates and stimulation assay performed by co-cultivating recombinant autologous DCs and autologous $\mathrm{CD} 3+$ cells.

\section{Isolation of CD3+ cells}

PBMCs were isolated from blood of healthy donors by density gradient centrifugation as explained above. $\mathrm{CD} 3+\mathrm{T}$ cells were isolated from PBMCs using Pan T Cell Isolation Kit II (Miltenyi Biotec, Bergisch Gladbach, Germany) and following the protocol from the supplier. In brief, PBMCs were washed with MACS buffer (Miltenyi Biotec, Bergisch Gladbach, Germany) and centrifuged at 1,200 rpm. Cell numbers were determined and the cells were incubated with biotin-antibody-cocktail (10 $\mu \mathrm{l} / 10^{7}$ cells) for $10 \mathrm{~min}$ at $4^{\circ} \mathrm{C} .30 \mu \mathrm{l}$ of MACS buffer and $20 \mu \mathrm{l}$ of anti-biotin-microbeads were added per $10^{7}$ cells and incub ated for 15 min at $4^{\circ} \mathrm{C}$. The cells were washed, centrifuged at $1,200 \mathrm{rpm}$ and the pellet was resuspended in MACS buffer. The labeled cells were passed through a magnetic separation column and flow through containing the CD3+ cells were resuspended in RPMI (Invitrogen) supplemented with $10 \%$ human $\mathrm{AB}$ sera (c.c.pro). These $\mathrm{CD} 3+\mathrm{T}$ cells were matured to CD8+ T cells and used further as effector cells in ACT assays and for analysis of $\mathrm{T}$-cell receptor $\mathrm{V} \beta$ repertoire.

\section{Stimulation assay}

Freshly transduced DCs were used to for the stimulation of autologous $\mathrm{CD} 3+\mathrm{T}$-cells isolated from HLA-B ${ }^{\star} 44$ :02 expressing PBMC donor. $1 \times 10^{5} \mathrm{DCs} / 200 \mu \mathrm{l}$ RPMI/10\% human AB-sera (Invitrogen; c.c.pro) were cultivated with $5 \times 10^{5} \mathrm{~T}$ cells overnight. 24 h post-stimulation, IL-7 (10 ng/ml) (PeproTech GmbH, Hamburg, Germany); IL-12 (0,1 ng/ml) (PeproTech GmbH, Hamburg, Germany); IL-15 (5 ng/ml) (PeproTech GmbH, Hamburg, Germany) in $50 \mu \mathrm{l}$ RPMI / $10 \%$ human AB-sera were added to the stimulation assay. Media was exchanged on day 4 and fresh RPMI / 10\% human $\mathrm{AB}$-sera supplemented with the interleukins was added to the assay.

\section{Flow cytometric analysis}

$\mathrm{T}$ cell responses were determined using the antibodies CD3/FITC, CD8/PE, CD4/PerCP (Beckman Coulter GmbH, Krefeld, Germany), CD45RA/APC and CD45RO/Pe-Cy7 (BD Biosciences, Heidelberg, Germany). Cells were washed twice in PBS, $0.5 \%$ BSA, $10 \mu \mathrm{l}$ of each antibody was added to the cells, incubated for $20 \mathrm{~min}$ at $4^{\circ} \mathrm{C}$, washed twice and measured using FacsCanto (BD Biosciences).

\section{ACT assay - Assay for cytotoxicity}

For the ACT assay, target cells were stained with CFSE (CarboxyFluorescein Diacetat Succinimidyl Ester) (Invitrogen) to a final concentration $2 \mu \mathrm{M}$. The reaction was stopped $3 \mathrm{~min}$ post-labeling and the cells were washed with PBS. Effector cells (stimulated CD8+ T-cells) were counted and the cytotoxicity assay was set up with a target: effector ratio $1: 10$. The cells were incubated for $24 \mathrm{~h}$ at $37^{\circ} \mathrm{C}$, washed and incubated with 7-aminoactinomycin (7-AAD) (Life Technologies, $\mathrm{GmbH}$. Germany). Percentage of cytotoxic lysis was measured by flow cytometry using FacsCanto and analysed using FlowJo. 


\section{Analysis of T-cell receptor $\mathrm{V} \beta$ repertoire}

T-cell receptor (TCR) V $\beta$ gene expression was assessed using TCRExpress ${ }^{\mathrm{TM}}$ Clonality Detecting Kit according to manufacturer's protocol (BioMed Immunotech, Alachua, FL). In brief, naïve CD3+ $\mathrm{T}$ cell population were co-cultured with autologous dendritic cells expressing recombinant HLA-B ${ }^{\star} 44: 02$ or $\mathrm{B}^{\star} 44: 03$ or $\mathrm{B}^{\star} 44: 35$ allele for 10 days. On day 10, RNA was extracted from $5^{\star} 10^{6} \mathrm{CTL}$ clones, following which cDNA was synthesized and the TCR genes were amplified in a single step of 35 PCR cycles. Specific TCR genes were amplified using TCR V $\beta$ gene-specific oligonucleotides by nested PCR for an additional 25 cycles. PCR products were resolved on a $4 \%$ high resolution agarose gel. The banding patterns were assessed and the $\mathrm{T}$ cell clonality was determined.

\section{Large-scale production of soluble HLA (sHLA) molecules and affinity purification}

The efficiency of transduced B-LCLs to produce sHLA-B ${ }^{\star} 44: 03$ and $-B^{\star} 44: 35$ molecules was analyzed by sandwich ELISA using w6/32 (Serotec, Düsseldorf, Germany) monoclonal antibody ( $\mathrm{mAb}$ ) as capture antibody. sHLA producing clones were grown in bioreactors CELLine (Integra, Fernwald, Germany). The harvests were collected from the bioreactors and centrifuged for $20 \mathrm{~min}$ at 1,200 rpm to remove cellular debris, filtered through a $0.45 \mu \mathrm{m}$ filter (Sartorius, Göttingen, Germany) and stored at $-20^{\circ} \mathrm{C}$. The supernatants were thawed, adjusted to $\mathrm{pH}$ 8.0 and affinity purified using N-hydroxysuccimide (NHS) - activated HiTrap columns coupled to mAb W6/32, using a BioLogic DuoFlow System (Bio-Rad, Hercules, USA). Trimeric complexes (class I heavy chain, $\beta_{2} \mathrm{~m}$ and peptide) were eluted using $0.1 \mathrm{M}$ glycine / $\mathrm{HCl}$ buffer ( $\mathrm{pH}$ 2.7) (Figure 3).

\section{Characterization of HLA-B ${ }^{\star} 44$-derived peptides}

Peptides obtained from sHLA-B ${ }^{\star} 44$ complexes from $2 \mathrm{mg}$ of total protein were differentiated into low and high binding peptides. The trimeric elution fractions were filtered through a $10 \mathrm{kDa}$ MWCO membrane (Millipore, Schwalbach, Germany) and the peptides detected in the flow-through were considered to be low binding. The retentate containing HLA- ${ }^{\star} 44$ trimeric complexes were then treated with $0.1 \%$ trifluoroacetic acid (TFA) to elute high binding peptides. Peptides were separated by filtering through a $10 \mathrm{kD}$ MWCO YM membrane (Millipore). Flow through fractions containing the low or high affinity peptides were subjected to Mass Spectrometry using an Eksigent nano-LC Ultra 2D HPLC coupled to an Orbitrap ion trap (Thermo Fischer, Waltham, Massachusetts, USA) providing a very high mass accuracy ( $<5 \mathrm{ppm})$. Mascot software [13] incorporating the IPI human and the respective decoy databases was used to carry out database queries.

\section{Molecular modelling}

Modelling of the $\mathrm{B}^{\star} 44: 35^{156 \mathrm{Glu}}$ allele was carried out with the program DeepView [14] using the available $\mathrm{B}^{\star} 44: 02^{156 \text { Asp }}$ structure (PDB 1M6O) [15] as a template and mutating 156 Asp to $156 \mathrm{Glu}$ using the internal rotamer library to find the best side chain orientations with minimum steric clashes. Each model was then subjected to energy minimization as implemented in DeepView. The graphics program PyMOL (http:// www.pymol.org) was used to generate all structure figures.

\section{Results}

T-cell priming using autologous DCs expressing recombinant allogeneic HLA-B ${ }^{\star} 44$ molecule

Monocyte derived dendritic cells (DCs) expressing HLA-B ${ }^{\star} 44: 02$ allele were transduced with lentiviral constructs encoding for HLA-B ${ }^{\star} 44: 03$ or $B^{\star} 44: 35$ allelic variants and these cells were subsequently used for the stimulation of freshly isolated autologous CD3+ T cells. Phenotypic changes of stimulated CD3+ T cells were monitored by flow cytometry. The cells were stained with CD45-RAPE and CD-45-RO-PE-Cy-5 antibodies and were gated for CD8+/ CD45RA+/CD45RO-, CD8+/CD45RA+/CD45RO+ and CD8+/ CD45RA-/CD45RO+ subsets. Activation of $\mathrm{T}$ cells by stimulation with recombinant autologous DCs expressing additional HLA-B ${ }^{\star} 44$ variant resulted in the loss of naïve T cell marker CD45-RA and gain of CD45-R0 expression among these cells (Figures 4a-4c). Stimulated CD8+ T cells also gained the expression of activation marker CD69 by day 8 (Figure $4 \mathrm{e}$ ).

\section{Cytotoxicity assay to prove functionality and specificity of stimulated CD8+ T-cells}

Cytotoxicity assays were performed using stimulated CD8+ T-cells as effector cells and recombinant autologous DCs expressing additional HLA-B ${ }^{\star} 44 / 156$ variant as target cells (Figure 5). Percentage of cytotoxic lysis was determined by labelling the target cells with CFSE and gating for CFSE-7AAD double positive populations. As controls, DCs from HLA- $B^{\star} 44: 02$ donors expressing additional $B^{\star} 44: 02$ or $B^{\star} 44: 03$ or $B^{\star} 44: 35$ allele were used as target cells and co-incubated with freshly isolated autologous CD3 + T cells as effector cells (target:effector ratio of 1:10) for $24 \mathrm{~h}$. Only 10\% target cells could be lysed by freshly isolated autologous CD3+ T cells ( $B^{\star} 44: 02$ specific) (Figure $\left.5 a-5 c\right)$. Cytotoxic activity of $\mathrm{CD} 8+\mathrm{T}$ cells $\left(\mathrm{B}^{\star} 44: 02\right.$ specific) stimulated with autologous DCs expressing additional $\mathrm{B}^{\star} 44: 02$ or $\mathrm{B}^{\star} 44: 03$ or $\mathrm{B}^{\star} 44: 35$ allele was determined by their ability to lyse specific recombinant $B^{\star} 44: 02$ or $\mathrm{B}^{\star} 44: 03$ or $\mathrm{B}^{\star} 44: 35$ expressing target $\mathrm{DCs}$ respectively. We found a significant percentage of $B^{\star} 44: 03$ (21\%) and $B^{\star} 44: 35$ (30\%) expressing target DCs to be lysed by $B^{\star} 44: 02$ specific CD8+ T cells (Figures $5 d-5 f$ ).

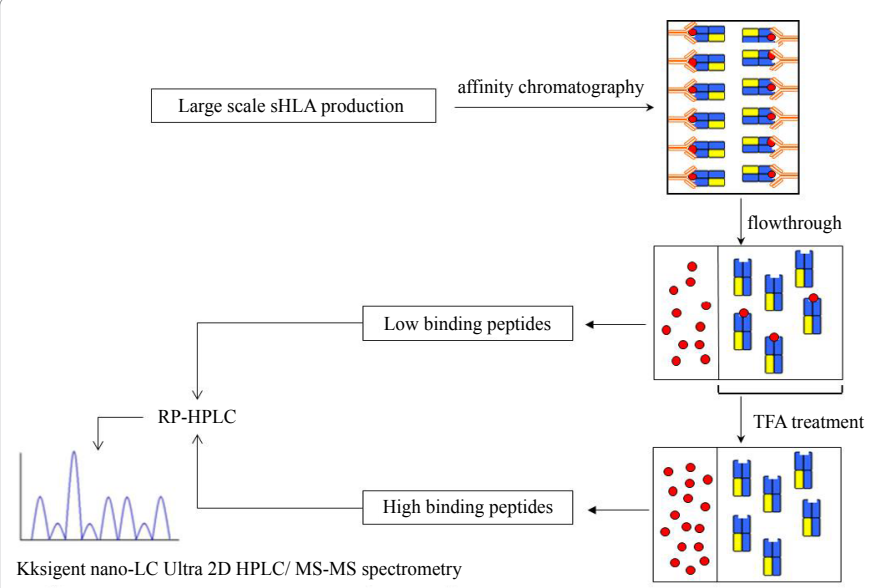

Figure 3: Engineering soluble HLA-B*44 molecules and peptide sequencing. Schematic overview of the steps involved in the generation of soluble HLA-B*44 molecules. SHLA-B*44 molecules were generated by stable lentiviral transduction of B-LCLs to express these molecules, followed by large scale production of these cells in bioreactors. Supernatant containing SHLA molecules were purified by affinity chromatography using mAb w6/32. Trimeric complexes were eluted using $0.1 \mathrm{M}$ glycine / HCl buffer $(\mathrm{pH} 2.7)$ Elution fractions were filtered through a $10 \mathrm{kDa}$ MWCO membrane and the peptides detected in the flow-through were considered to be low binding ones. The retentate containing trimeric complexes were then treated with $0.1 \%$ trifluoroacetic acid (TFA) to elute high binding peptides. Peptides were purified by RP-HPLC and subjected to mass spectrometry using an Eksigent nano-LC Ultra 2D HPLC coupled to an Orbitrap ion trap. 

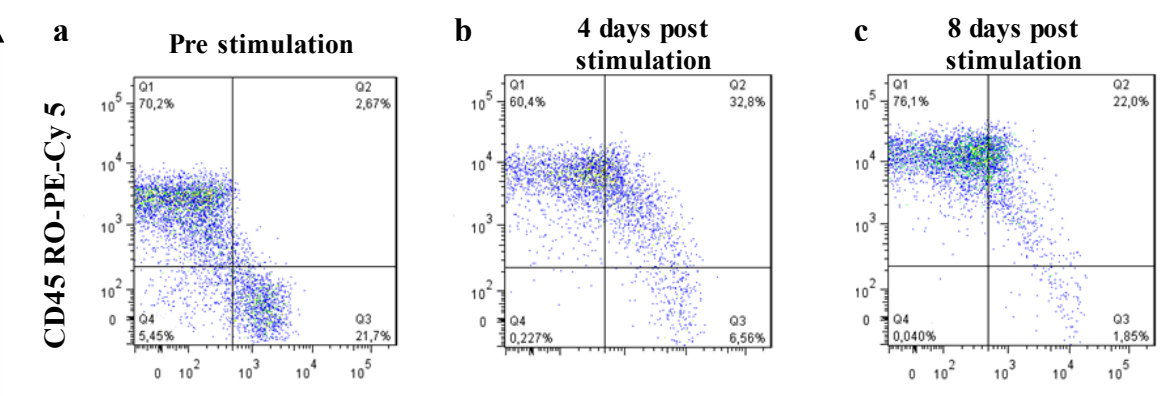

CD45-RA PE

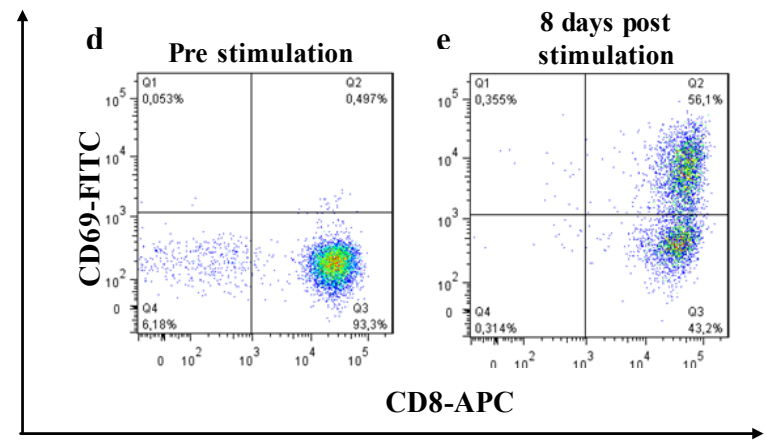

Figure 4: Phenotypic analysis of stimulated CD8+ T-cells from a HLA-B*44:02 positive donor. Representative FACS plots of the phenotypic analysis of stimulated CD8+ T-cells from an HLA-B*44:02 positive donor. CD3+ T cells isolated from human peripheral blood were stimulated with autologous $\mathrm{DCs}$ expressing additional recombinant HLA-B*44/ 156 variant. The cells were stained with CD45-RA-PE and CD-45-RO-PE-Cy-5 antibodies on day 0 , 4 or 8 , respectively, and were gated for CD8+/CD45RA+/CD45RO-, CD8+/CD45RA+/CD45RO+ and CD8+/CD45RA-/CD45RO+ subsets. Activation resulted in loss of CD45-RA and gain of CD45-R0 expression in these cells $(4 a, b, c)$. In addition, stimulated CD8+ T cells gained the expression of CD69 activation marker (Figure 4e).
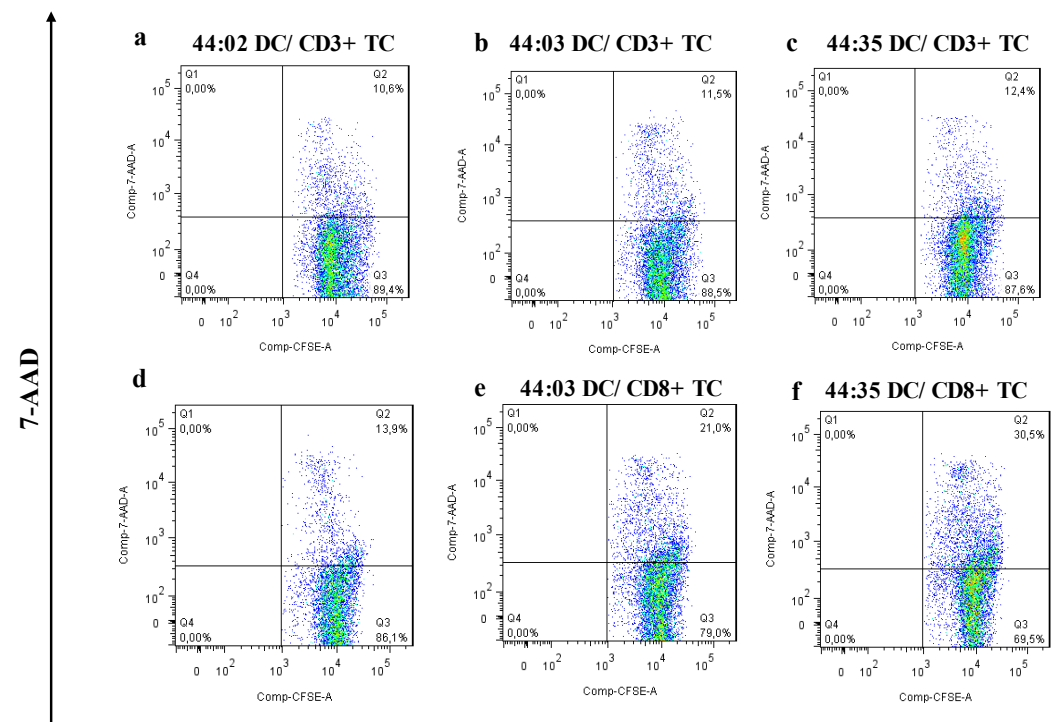

CFSE

Figure 5: Antigen specific CD8+ CTL assay using recombinant DCs expressing an artificial HLA-B*44:03 or B*44:35 molecules as target. Antigen-specific CD8+ CTL activity against the allogeneic HLA-B*44/156 variants was determined by flow cytometry. Target DCs transduced with lentiviral constructs to express additional HLA-B*44/ 156 variant were labelled with CFSE and lysis of these cells by CTLs was determined by staining with 7-AAD.

$5 a, b, c)$ neg. controls for antigen specific CTL response. Freshly isolated CD3+ T cells from an HLA-B*44:02 expressing donor were incubated with autologous DCs expressing additional HLA-B*44/156 molecule - i.e $B^{*} 44: 02$ or $B^{*} 44: 03$ or $B^{*} 44: 35$.

$5 d$, e, f) CD8+ T cells stimulated with autologous DCs expressing additional $B^{\star} 44: 02$ or $B^{*} 44: 03$ or $B^{*} 44: 35$ allele were used as effector cells against recombinant $B^{\star} 44: 02$ or $B^{\star} 44: 03$ or $B^{\star} 44: 35$ expressing target $D C s$ respectively. A high percentage of $B^{\star} 44: 03(21 \%)$ and $B^{\star} 44: 35(30 \%)$ expressing target $D C s$ were found to be lysed by autologous CD8+ T cells from the $B * 44: 02$ donor. 
Citation: Badrinath S, Huyton T, Kunze-Schumacher H, Elsner HA, Blasczyk R, et al. (2014) Differential Impact of HLA-B*44 Allelic Mismaches at Position 156 on Peptide Binding Specificities and T-Cell Diversity. J Stem Cell Res Ther 4: 192. doi:10.4172/2157-7633.1000192

Results of the cytotoxicity assay confirm specificity of TCRs which are selected by subtle alterations within the HLA molecules.

\section{Clonal diversity in the TCR V $\beta$ repertoire of CTLs cultured} with autologous DCs expressing recombinant HLA-B ${ }^{\star} 44 /$ 156 variants

Clonality pattern of the CTL clones cultured with autologous DCs expressing recombinant HLA-B ${ }^{\star} 44 / 156$ variants was investigated by RT-PCR of the TCR V $\beta$ gene families. Here, amplification of V $\beta$ genes from monoclonal or oligoclonal expanded $\mathrm{T}$ cells is indicated by a single or multiple defined bands. Amplification of $\mathrm{V} \beta$ genes from $\mathrm{T}$ cells of polyclonal origin display a smear with undefined bands.

We found differential expression of V $\beta$ genes; CTLs $\left(B^{\star} 44: 02\right.$ specific) cultured with autologous DCs expressing recombinant $B^{\star} 44: 02$ molecule displayed polyclonal pattern with smear bands in most of the TCR V $\beta$ gene families, implicating broader V $\beta$ repertoire (Figure 6a). In contrast, CTLs ( $B^{\star} 44: 02$ specific) cultured with autologous DCs expressing recombinant $\mathrm{B}^{\star} 44: 03$ or $\mathrm{B}^{\star} 44: 35$ molecule displayed monoclonal/ oligoclonal patterns as evident by the visibility of multiple clear bands or a single band for some of the $\mathrm{V} \beta$ gene families (Figure $6 b-B^{\star} 44: 03 V \beta 6,11,23$ and Figure $\left.6 c-B^{\star} 44: 35 V \beta 11,12,14,15,16\right)$.

\section{Peptide features}

Outcome following transplantation is majorly influenced by $\mathrm{T}$ cell recognition of class I alleles bound to peptides with distinct amino acid sequences as self/ antigenic. We used peptide sequencing as a means to determine if polymorphic differences occurring at position 156 in $B \star 44: 03$ or $B \star 44: 35$ alleles influenced the peptide binding specificities of these molecules by comparing the peptides eluted from them with $B^{\star} 44$ :02 derived peptides published earlier [9]. A total of $2 \mathrm{mg}$ of Peptides derived from sHLA-B ${ }^{\star} 44 / 156$ variants were classified into low and high binding peptides based on the criteria metioned in the materials and methods section. Peptides were purified by RP-HPLC and sequenced using Eksigent nano-LC Ultra 2D HPLC coupled to an Orbitrap ion trap providing a very high mass accuracy.

The profile of peptides recovered from $B^{\star} 44: 35$ molecules showed 24 peptides that were low binding and 56 high binding peptides. Interestingly, we were not able to recover any low binding peptides from $B^{\star} 44: 03$. However, upon TFA treatment, we were able to identify 38 peptides of high affinity.

Peptides eluted from sHLA-B ${ }^{\star} 44: 35$ demonstrate a strong shift in the binding preference at the peptide $\mathrm{C}$ terminus

Peptides eluted from sHLA-B ${ }^{\star} 44$ :02 or $B^{\star} 44$ :03 demonstrated exclusively Glu at position 2 , while $B^{\star} 44: 35$-derived peptides exhibited a small fraction of other residues such as Lys/ Leu in addition to Glu based on the frequency of residues at this position (Figures $7 \mathrm{a}$ and $7 \mathrm{c}$ ). Major anchor residues at the $\mathrm{C}$ terminus among the peptides derived from $B^{\star} 44: 02$ was found to be $\operatorname{Trp}(\sim 45 \%)$ or Tyr $(\sim 25 \%)$ while $B^{\star} 44: 03$ demonstrated higher preference for Tyr (38\%), followed by Phe $(36 \%)$ at this position and only a small percentage of Trp (9\%) (Figures $7 \mathrm{~b}$ and $7 \mathrm{~d}$ ). We observed a strong shift in binding prefence at the $\mathrm{C}$ terminus among the peptides eluted from $\mathrm{B}^{\star} 44: 35$. Leu (26\%), Lys (26\%) and $\operatorname{Trp}(22 \%)$ were found to occur most frequently at the $C$ terminus among the low binding peptides recovered from this allotype (Figure $7 b$ ). Among the high binding- ${ }^{\star} 44$ :35-derived peptides, Lys (51\%) was found to be the major anchor residue at the $\mathrm{C}$ terminus, followed by Tyr (24\%) and Phe (20\%) (Figure 7d). Also, Lys at C

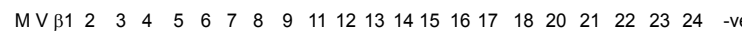

a
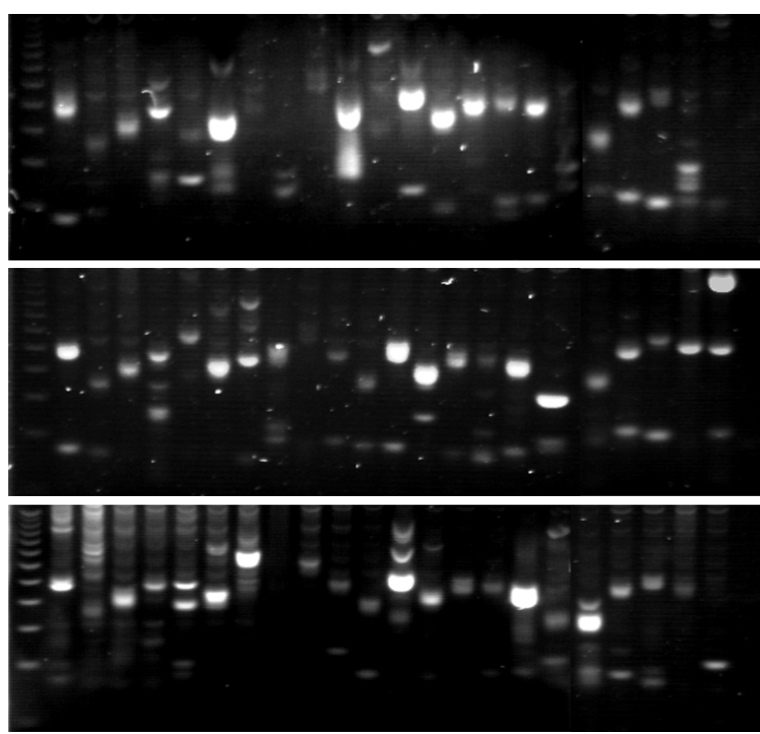

Figure 6: Expression patterns of TCR V $\beta$ genes in CTLs co-cultured with autologous DCs expressing recombinant $B^{*} 44 / 156$ variants. RNA was extracted from CTLs co-cultured with autologous DCs expressing recombinant $B * 44 / 156$ variants, following which cDNA was synthesized and specific TCR $V \beta$ genes were amplified by nested PCR. We found differential patterns in the expression of $V \beta$ genes among $B * 44: 02$ specific $C T L s$ cultured in the presence of autologous DCs expressing $B^{*} 44: 02(6 a), B^{*} 44: 03(6 b)$ and $B^{*} 44: 35(6 c)$.

$\mathrm{M}, 10 \mathrm{bp}$ ladder; -ve, negative control; V $\beta 1-24$ - TCR V $\beta$ subfamilies.

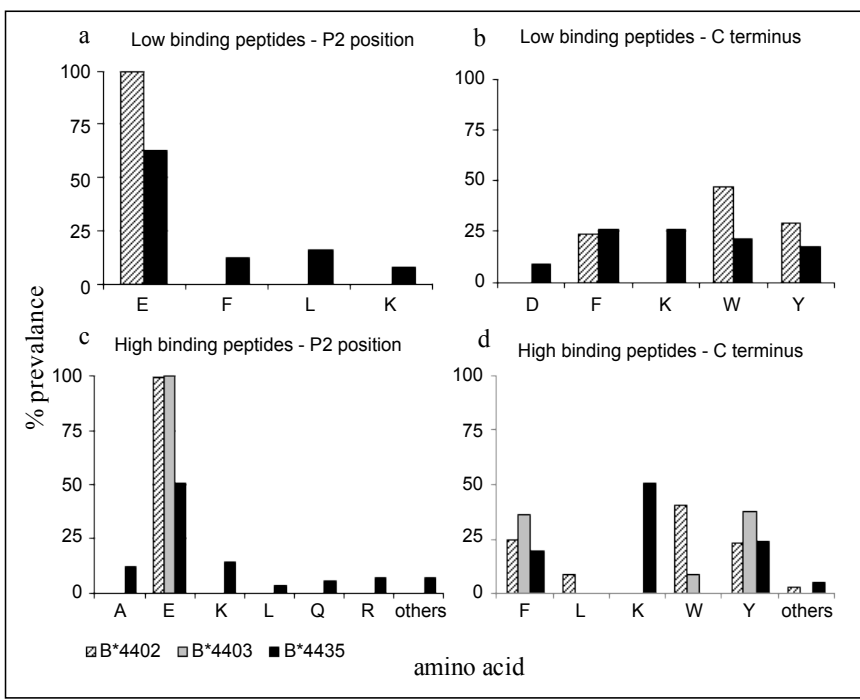

Figure 7: Amino acid frequencies at $\mathrm{p} 2$ and at $\mathrm{C}$ terminus of peptides eluted from $s B^{*} 44 / 156$ variants. $A A$ frequencies occurring at peptide position 2 and at $C$ terminus among the peptides eluted from sHLA-B*44/ 156 variants. The $A A$ residues are plotted on the $X$-axis and the percentage prevalence of each of these residues is plotted on the $\mathrm{Y}$-axis. Crossed bars, grey bars and black bars represent SHLA-B*44:02, $B^{*} 44: 03$ and $B * 44: 35$, respectively. No low binding peptides were recovered from $B^{*} 44: 03$. Both $B^{*} 44: 02$ and $B^{*} 44: 03$ bound exclusively Glu at peptide position $2(P 2)$ unlike $B^{*} 44: 35$ which bound a small percentage of other residues - Ala (only in high binding peptides), Lys and Leu. Among the low and high binding peptides eluted from HLA-B*44:02 and $B * 44: 03$, Trp $>\operatorname{Ty} \geq \geq$ Phe were found to occur more frequently at the $C$ terminus. Lys at $C$ terminus was exclusive to peptides eluted from $B^{*} 44: 35$. 
teminus was unique to peptides eluted from $\mathrm{B}^{\star} 44: 35$ and was absent in peptides derived from the other two allotypes.

\section{sHLA-B ${ }^{\star} 44: 35$ alleleic variant presents peptides of extra- ordinary length}

Comparison of peptides lengths demonstrated that the low binding peptides eluted from sHLA-B ${ }^{\star} 44: 02$ were all of canonical length, with nona $(23 \%)$ and decamer $(64 \%)$ peptides occuring more frequently. In addition, this allotype was also found to present a small fraction of octamer peptides $(6 \%)$. In comparision, octamer peptides were absent among the low binding peptides eluted from $B^{\star} 44: 35$. We were able to identify a significant percentage of non-canonical peptides (24\%) amongst this peptide subset, in addition to peptides of canonical lengths (Figure 8a). Majority of the high binding peptides presented by $B^{\star} 44: 02$ and $B^{\star} 44: 03$ were of canonical length ( 90\%). In contrast, we found that $B^{\star} 44: 35$ variants presented a significant proportion $(\sim$ $40 \%$ ) of high binding peptides of extraordinary length, with the longest peptide containing up to 28 AAs (Figure $8 \mathrm{~b}$ ).

\section{A small percentage of the overall peptide repertoire is shared between the three different $B \star 44 / 156$ allotypes}

As the $B^{\star} 44 / 156$ variants were all expressed in the same cell line (B-LCL 721.221), their peptide sources were identical, although these subtypes were found to share only a small subset of their overall peptide repertoire (3\%). $\mathrm{B}^{\star} 44: 02$ was found to share $\sim 8 \%$ of its repertoire with peptides recovered from $B^{\star} 44: 03$ and $6 \%$ with $B^{\star} 44: 35$. We found a

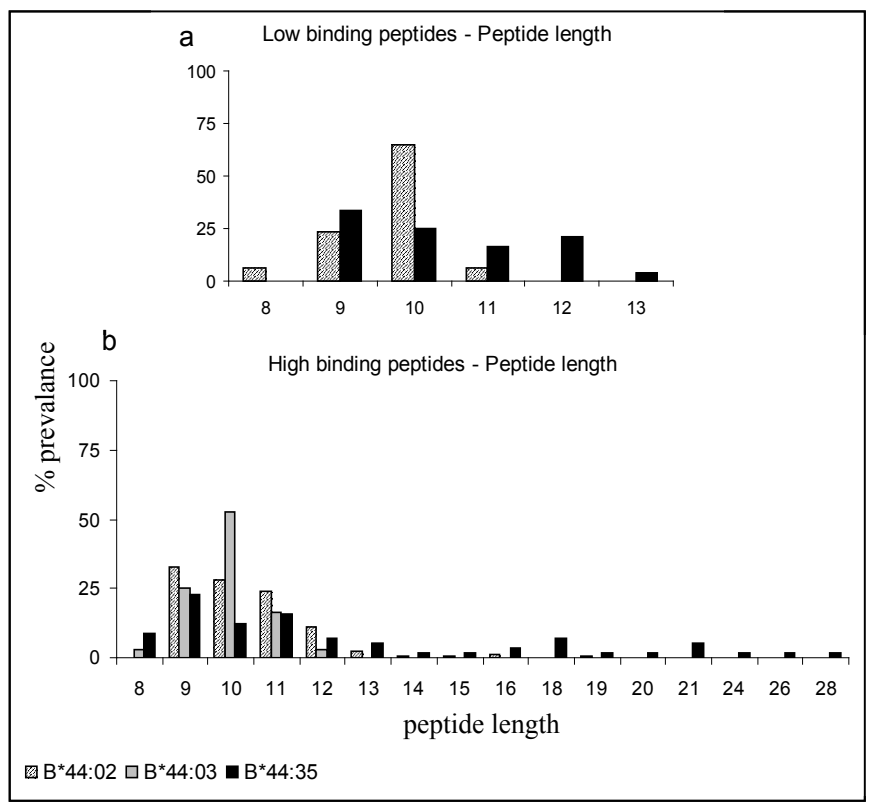

Figure 8: Length of peptides presented by $B * 44 / 156$ variants. This diagram indicates the length of low and high binding peptides eluted from different HLA-B*44/ 156 variants. Peptide length is plotted on the X-axis and the percentage prevalence of peptides of a particular length is plotted on the $Y$ axis. Crossed, grey and black bars represent values from HLA-B*44:02, $B^{*} 44: 03$ and $B^{*} 44: 35$, respectively. There were no low binding peptides eluted from $B^{*} 44: 03$. The low binding peptides presented by HLA-B*44:02 were all found to be of canonical length (8-11 AAs), a significant percentage of low binding peptides presented by $B^{*} 44: 35$ was found to be longer than canonical length $(24 \%)$. Majority of the high binding peptides presented by $B^{*} 44: 02$ and $B^{*} 44: 03$ were of canonical length $(\sim 90 \%)$. $B^{*} 44: 35$ variants were found to present a significantly high percentage $(\sim 40 \%)$ of high binding peptides of extraordinary length, with the longest peptide containing up to $28 \mathrm{AAs}$. slightly higher percentage of overlapping peptide repertoire shared between the $B^{\star} 44: 03$ and $B^{\star} 44: 35$ subtypes (11\%) (Figure 9).

\section{Molecular modelling of the $B^{\star} 44: 35^{156 G l u}$ micropolymorphism}

Our previously described bioinformatic tool "Pocketcheck" [8] is a database of contact data for deposited HLA-class I crystal structures and highlights those heavy chain residues that frequently contact particular peptide residue positions [8]. From these data it is clear that position 156 can contact positions 3, 4, 5, 6 and/or 7 of a bound peptide but is unable to directly contact the C-terminal $(P \Omega)$ position of a peptide. Since peptide sequencing from the $B^{\star} 44: 35^{156 \mathrm{Glu}}$ allele caused an unexpected change in the $P \Omega$ anchor compared to $B^{\star} 44: 02^{156 \text { Asp }}$ we generated a molecular model of the $\mathrm{B}^{\star} 44: 35^{156 \mathrm{Glu}}$ allele to understand the altered molecular features now selecting Lys at this position.

The substitution of $A s p\left(B^{\star} 44: 02\right)$ with Glu $\left(B^{\star} 44: 35\right)$ at position 156 in our modelling generated a steric clash between 156Glu and 114Asp, suggesting that 114Asp is forced to adopt an alternate conformation (Figure 10). This subtle change appears to ripple across the floor of the peptide binding groove and we predict that the effect manifests itself by disrupting the contact between 114Asp and 116Asp that is partially mediated through an active water molecule. The resulting geometry of 116Asp now suggests a more favourable orientation to bind peptides containing a C-terminal Lys residue.

\section{Discussion}

Donor selection process during HSCT involves rigorous assessment and matching of HLA types between the donor:recipient pair [3]. The best clinical results following transplantation have been achieved when HLA matched sibling donor is used for transplantation. However, there is only a $30 \%$ chance of finding such a donor and in most instances, stem cells from the most compatible HLAmismatched related/ unrelated donor are used for transplantation [1]. Polymorphisms occurring in HLA class I molecules determine peptide selection and thus the conformation of the whole pHLA complex. The effect of individual polymorphisms remains poorly understood due to discrepancies arising post-transplantation [16,17]. Therefore, it is of crucial importance to determine the effect of every individual amino acid (AA) polymorphism occurring within the class I molecules to evaluate the best permissible mismatches in order to reduce/avoid post-transplant complications.

To date, there is an increase in the number of stem cell donors and availability of high resolution four-digit HLA typing, supporting the chance to find a well matched donor: recipient pair. However, there is currently no strategy to determine the allogenicity of selected single AA exchanges to support clinical decisions during a transplantation scenario. If several donors, each with different HLA class I mismatches are available, the relative alloreactivity between different mismatches cannot be predicted due to lack of adequate in vitro and in vivo data for ranking mismatch effect. Therefore, we aim to resolve this issue by integrating the observed allogeneic $\mathrm{T}$ cell responses in vitro along with the peptide sequencing data to determine if a particular mismatch might be acceptable for transplantation. Our artificial allogeneic system provides a tool to distinguish the effects of mHAGs from those of HLA mismatches. In this system, observed allogeneic $\mathrm{T}$ cell responses can be attributed exclusively due to the specific mismatched AA between the donor and recipient HLA class I molecule. In order to validate our artificial allogeneic system, we chose the HLA-B ${ }^{\star} 44$ group as it is most commonly occurring group in the Caucasian population and contains allelic subtypes with single AA mismatches occurring at 


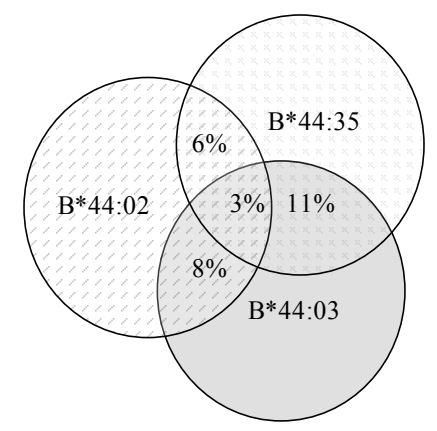

Figure 9: Shared peptide repertoire between the HLA-B*44/ 156 variants. This Venn diagram represents shared peptide percentage between HLA-B*44:02, B*44:03 and $B * 44: 35$ allelic variants. $3 \%$ of the overall peptide repertoire was found to be shared between the three different allotypes. Of the peptides eluted from $B * 44: 02,8 \%$ of its repertoire overlapped with $B * 44: 03$ peptides and $6 \%$ of it with $B * 44: 35$. A slightly higher percentage of the peptide repertoire was found to be shared between the $B^{*} 44: 03$ and $B^{*} 44: 35$ allotypes.
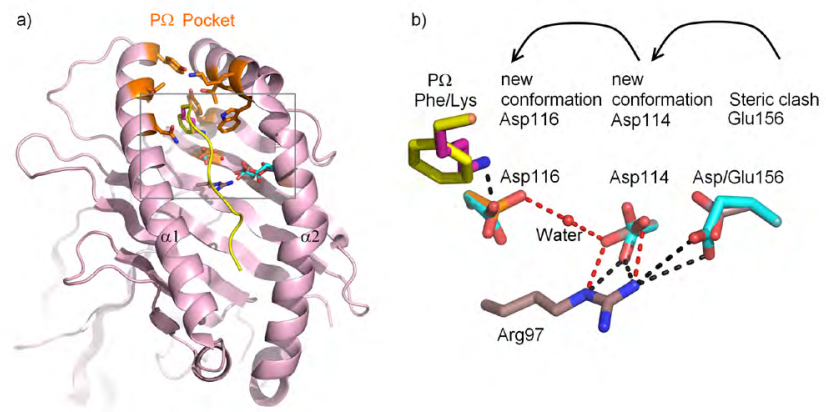

Figure 10: Molecular modelling of the HLA-B*44:35156Glu micropolymorphism. 10a) Structural comparison of the HLA-B*44:02156Asp and HLA-B*44:35156Glu variants. A view is shown of the peptide binding groove with the HLA heavy chain represented as ribbons. Highlighted as sticks is the $p \Omega$ peptide anchor (yellow/ dark pink), the residues comprising the $p \Omega$ binding pocket (orange), residues 156Glu, 114Asp and 116Asp from the HLAB*44:35156Glu model are coloured cyan.

b) A close up view of the molecular details depicted in a), the steric clash by introducing $156 \mathrm{Glu}$ disrupts the $97 \mathrm{Arg}-114 \mathrm{Asp}$-water-116Asp hydrogen bonding network (red dashes) by forcing a conformational change in 114Asp. 116 Asp is then more likely to be in a receptive conformation to accept Lys as a $\mathrm{p} \Omega$ anchor residue.

position 156 within the $\alpha 2$ helix of HLA class I heavy chain. AA at position 156 is regarded to be critical in the donor selection process because mismatches at this position are found to cause alloreactive $\mathrm{T}$ cell responses $[10,18]$. Within the HLA-B ${ }^{\star} 35$ group, it was observed that the HLA-B ${ }^{\star} 35: 01^{156 \text { Leu }}$ versus $B^{\star} 35: 08^{156 \text { Arg }}$ mismatch elicited strong cytotoxic $\mathrm{T}$ cell responses, most likely due to conformational changes occurring within the peptide binding region (PBR) [19]. This conformational change alters the peptide-binding affinity, resulting in presentation of peptides with allele-specific epitopes differing both in their lengths and immunogenicity [20]. Within the $B^{\star} 44$ group it was shown that mismatching HLA-B ${ }^{\star} 44: 02^{156 \text { Asp }}$ with $B^{\star} 44: 03^{156 \text { Leu }}$ was found to result in transplant rejection and acute GvHD, indicating that they represent significant barriers to clinical transplantation [10]. Another naturally occurring polymorphism within this allelic group is $\mathrm{B}^{\star} 44: 35^{156 \mathrm{Glu}}$ that shares the same nature of polar, AA group at position 156. We wanted to determine if this AA exchange would be permissible in a transplantation setting using our artificial allogeneic system system. In this system, donor DCs expressing HLA-B ${ }^{\star} 44: 02$ allele were transduced with additional full length $B^{\star} 44: 35$ molecule and these cells were subsequently co-incubated with autologous CD3 $+\mathrm{T}$ cell isolated from the same $\mathrm{B}^{\star} 44: 02$ donor. Stimulated T cells were used in cytotoxicity assay to monitor if they elicited specific $T$ cell responses against the allogeneic $\mathrm{B}^{\star} 44$ :35 expressing DCs and the percentage of target cell lysis were used as a measure of allogenicity. To elucidate the accuracy of our in vitro system, we also examined the $\mathrm{T}$ cells responses of $\mathrm{B}^{\star} 44: 02$ towards autologous DCs expressing recombinant $B^{\star} 44: 03$, as this particular transplantation direction was shown to elicit highly allogeneic responses in a clinical transplantation scenario [10]. The alloreactivity of $B^{\star} 44: 02$ specific CD8+ $T$ cells towards $B^{\star} 44: 35$ (30\%) targets was higher than towards $B^{\star} 44: 03$ (21\%), while only $13 \%$ of $B^{\star} 44: 02$ expressing target cells were lysed, indicating specificity of TCRs which are selected by subtle alterations within the HLA molecules. Our artificial allogeneic system provides a basis for systematic analysis of individual mismatches and predicts host cell permissively for each mismatch combination accurately. The higher the dissimilarity between two mismatched AA, greater would be the risk of T-cell-mediated immune response.

As outcome following HSCT is to a large extent influenced by the $\mathrm{T}$ cell recognition of peptides bound to HLA class I molecules as self/ allogeneic [11], histocompatibility can further be defined based on the features and repertoire of peptides presented by specific allotypes. We wanted to investigate if the observed allogenicity between the $\mathrm{B}^{\star} 44 /$ 156 variants reflected on the peptides presented by them. Although the majority of HLA alleles present peptides terminating in hydrophobic AAs the proteasome has at least three different catalytic activities: trypsin-like (i.e. cleavage after basic AAs), chemotrypsin-like (i.e. cleavage after large, hydrophobic AAs) and peptidyl-glutamyl-peptide hydrolyzing activity (i.e. cleavage after acidic AAs). Here we used soluble HLA technology and investigated the endogenous peptides presented by $B^{\star} 44: 02, B^{\star} 44: 03$ and $B^{\star} 44: 35$ allotypes to determine if there are any significant alterations in the binding motifs and lengths in peptides presented by them. We found only $3 \%$ of the overall peptide repertoire to be shared between these allotypes. Systematic characterization of the ligands derived from the $\mathrm{B}^{\star} 44$ allotypes demonstrated that the $\mathrm{p} 2$ position in $B^{\star} 44: 02$ and $B^{\star} 44: 03$ was conserved and exclusively Glu occured at this position while in $\mathrm{B}^{\star} 44: 35$, other residues such as Lys/ Leu also occured in addition to Glu. Our peptide elution data derived from $\mathrm{B}^{\star} 44$ :35 highlighted a strong shift in the binding preference for Lys at the $\mathrm{C}$ terminus, while this residue was completely absent in peptides eluted from $B^{\star} 44: 02$ and $B^{\star} 44: 03$.

The observation of such a change in the peptide anchor in $B^{\star} 44: 35$ as a result of a highly conserved substitution at position 156 was unexpected. Our molecular modelling of the $\mathrm{B}^{\star} 44: 35$ structure has however allowed us to postulate how this may be achieved and subtle differences in the conformations and energetics of the "floor" of the peptide binding grove clearly influence the ability of individual alleles to select specific peptide repertoires. The selection of Lys as a $\mathrm{P}$ anchor is relatively uncommon, compared to many other alleles, however the binding of peptides with Lys at the C terminus (27\%) versus its complete absence has been demostrated for the ankylosing spondylytis (Bechterew's disease) which is associated with $\mathrm{B}^{\star} 27: 05^{116 \mathrm{Asp}}$ but not with its protective counterpart $B^{\star} 27: 09^{116 H i s}$. Additionally it has been demonstrated in $B^{\star} 27: 05$ and $B^{\star} 27: 09$ alleles that the binding of peptides containing a Lys $P \Omega$ anchor appears to be weaker and thermodynamically less stable [21]. Such allosteric mechanisms might influence not only the association and dissociation of the trimeric complexes, but also the mode of recognition by the $\mathrm{T}$-cell receptors [22] 
Analysis of the peptide binding affinities showed that there were no peptides of low affinity that could be recovered from the $\mathrm{B}^{\star} 44: 03$ molecules, indicating a role for Leu at 156 in increasing the stability of the peptide-HLA complex. Although the binding affinity of the peptide is not indicative of the relative immunogenicity of the peptide, higher affinity peptides generally extend the half-life of the peptideHLA complex at the cell surface, thereby increasing the time available for T-cell receptor recognition. In addition to variations in the binding motifs and binding affinities, we also found marked differences in the length of peptides presented by these allotypes. The majority of pepides presented by $\mathrm{B}^{\star} 44: 02$ and $\mathrm{B}^{\star} 44: 03$ were 9-11 AAs long, with a small fraction of octameric peptides. $B^{\star} 44: 35$ variants on the other hand were found to present a significantly high number of peptides of extraordinary length, accounting for $\sim 47 \%$ of peptides, with the longest peptide containing up to 28 AAs. In addition, HLA-B ${ }^{\star} 44: 35$ is found to disfavour the binding and presentation of octamer peptides.

These data highlight the need for direct comparison of HLA alleles at many levels in order to determine how permissive individual micropolymorphisms can be. Indeed it is clear that neither direct sequence comparison nor molecular modelling would have predicted the change in $P \Omega$ anchor motif observed by direct sequencing of the bound peptide repertoire. Only then we can see, using the detailed structures of HLA molecules, how such subtle conformational changes and why the manipulation of a few hydrogen bonds within a protein could ultimately be responsible for successful transplantation and patient survival.

\section{Acknowledgement}

The study was founded in part by the German Jose Carreras Foundation.

\section{References}

1. Zuckerman T, Rowe JM (2007) Alternative donor transplantation in acute myeloid leukemia: which source and when? Curr Opin Hematol 14: 152-161. [Pubmed].

2. Petersdorf EW (2007) Risk assessment in haematopoietic stem cell transplantation: histocompatibility. Best Pract Res Clin Haematol 20: 155-170. [Pubmed].

3. Petersdorf EW (2008) Optimal HLA matching in hematopoietic cell transplantation. Curr Opin Immunol 20: 588-593. [Pubmed].

4. Matzinger P, Bevan MJ (1977) Hypothesis: why do so many lymphocytes respond to major histocompatibility antigens? Cell Immunol 29: 1-5. [Pubmed].

5. Rammensee HG, Bevan MJ (1984) Evidence from in vitro studies that tolerance to self antigens is MHC-restricted. Nature 308: 741-744. [Pubmed].

6. Dierselhuis M, Goulmy E (2009) The relevance of minor histocompatibility antigens in solid organ transplantation. Curr Opin Organ Transplant 14: 419425. [Pubmed].

7. Matzinger $P$ (1997) The immune system's role in graft loss: theoretic considerations. Transplant Proc 29: 11S-12S. [Pubmed].
8. Huyton T, Ladas N, Schumacher H, Blasczyk R, Bade-Doeding C (2012) Pocketcheck: updating the HLA class I peptide specificity roadmap. Tissue Antigens 80: 239-248. [Pubmed]

9. Badrinath S, Saunders P, Huyton T, Aufderbeck S, Hiller O, et al. (2012) Position 156 influences the peptide repertoire and tapasin dependency of human leukocyte antigen $B^{*} 44$ allotypes. Haematologica 97: 98-106. [Pubmed].

10. Fleischhauer K, Kernan NA, O'Reilly RJ, Dupont B, Yang SY (1990) Bone marrow-allograft rejection by $T$ lymphocytes recognizing a single amino acid difference in HLA-B44. N Engl J Med 323: 1818-1822. [Pubmed].

11. Chelvanayagam G, Jakobsen IB, Gao X, Easteal S (1996) Structural comparison of major histocompatibility complex class I molecules and homology modelling of five distinct human leukocyte antigen-A alleles. Protein Eng 9: 1151-1164. [Pubmed].

12. Saper MA, Bjorkman PJ, Wiley DC (1991) Refined structure of the human histocompatibility antigen HLA-A2 at 2.6 A resolution. J Mol Biol 219: 277-319. [Pubmed]

13. Hirosawa M, Hoshida M, Ishikawa M, Toya T (1993) MASCOT: multiple alignment system for protein sequences based on three-way dynamic programming. Comput Appl Biosci 9: 161-167. [Pubmed]

14. Guex N, Peitsch MC (1997) SWISS-MODEL and the Swiss-PdbViewer: an environment for comparative protein modeling. Electrophoresis 18: 2714-2723. [Pubmed].

15. Macdonald WA, Purcell AW, Mifsud NA, Ely LK, Williams DS, et al. (2003) A naturally selected dimorphism within the HLA-B44 supertype alters class I structure, peptide repertoire, and T cell recognition. J Exp Med 198: 679-691. [Pubmed].

16. Mickelson EM, Petersdorf E, Anasetti C, Martin P, Woolfrey A, et al. (2000) HLA matching in hematopoietic cell transplantation. Hum Immunol 61: 92-100. [Pubmed].

17. Ottinger HD, Ferencik S, Beelen DW, Lindemann M, Peceny $R$, et al. (2003) Hematopoietic stem cell transplantation: contrasting the outcome of transplantations from HLA-identical siblings, partially HLA-mismatched related donors, and HLA-matched unrelated donors. Blood 102: 1131-1137. [Pubmed]

18. Fleischhauer K, Avila D, Vilbois F, Traversari C, Bordignon C, et al. (1994) Characterization of natural peptide ligands for HLA-B*4402 and $-B^{*} 4403$ : implications for peptide involvement in allorecognition of a single amino acid change in the HLA-B44 heavy chain. Tissue Antigens 44: 311-317. [Pubmed].

19. Tynan FE, Burrows SR, Buckle AM, Clements CS, Borg NA, et al. (2005) T cell receptor recognition of a 'super-bulged' major histocompatibility complex class I-bound peptide. Nat Immunol 6: 1114-1122. [Pubmed]

20. Tynan FE, Reid HH, Kjer-Nielsen L, Miles JJ, Wilce MC, et al. (2007) A $T$ cell receptor flattens a bulged antigenic peptide presented by a major histocompatibility complex class I molecule. Nat Immunol 8: 268-276. [Pubmed].

21. Hülsmeyer M, Welfle K, Pöhlmann T, Misselwitz R, Alexiev U, et al. (2005) Thermodynamic and structural equivalence of two HLA-B27 subtypes complexed with a self-peptide. J Mol Biol 346: 1367-1379. [Pubmed].

22. Nurzia E, Narzi D, Cauli A, Mathieu A, Tedeschi V, et al. (2012) Interaction pattern of Arg 62 in the A-pocket of differentially disease-associated HLA-B27 subtypes suggests distinct TCR binding modes. PLoS One 7: e32865. [Pubmed]. 